\title{
Interval-Valued Intuitionistic Fuzzy Einstein Geometric Choquet Integral Operator and Its Application to Multiattribute Group Decision-Making
}

\author{
Qifeng Wang $(\mathbb{D}$ and Haining Sun \\ Logistics and E-Commerce College, Zhejiang Wanli University, Ningbo 315100, China \\ Correspondence should be addressed to Qifeng Wang; lhywqf@163.com
}

Received 20 July 2017; Revised 4 November 2017; Accepted 7 November 2017; Published 14 January 2018

Academic Editor: Peide Liu

Copyright (C) 2018 Qifeng Wang and Haining Sun. This is an open access article distributed under the Creative Commons Attribution License, which permits unrestricted use, distribution, and reproduction in any medium, provided the original work is properly cited.

\begin{abstract}
With respect to the multiattribute decision-making (MADM) problem in which the attributes have interdependent or interactive phenomena under the interval-valued intuitionistic fuzzy environment, we propose a group decision-making approach based on the interval-valued intuitionistic fuzzy Einstein geometric Choquet integral operator (IVIFEGC). Firstly, the Einstein operational laws and some basic principle on interval-valued intuitionistic fuzzy sets are introduced. Then, the IVIFEGC is developed and some desirable properties of the operator are studied. Further, an approach to multiattribute group decision-making with interval-valued intuitionistic fuzzy information is developed, where the attributes have interdependent phenomena. Finally, an illustrative example is used to illustrate the developed approach.
\end{abstract}

\section{Introduction}

The intuitionistic fuzzy set (IFS) [1] is the generalization of fuzzy set theory proposed by Zadeh [2]. The IFS includes the membership degree, nonmembership degree, and hesitancy degree which is more flexible in dealing with the imprecise and vague information. Presently, the IFS has been widely used in many fields, such as machine learning, decisionmaking, and pattern recognition. Meanwhile, many experts study the IFS theory and have many achievements. Yu et al. [3-5] make some reviews on the development of the IFS. In many real decision-making problems, the decision-makers often have difficulties in determining the membership degree and nonmembership degree with the crisp numbers. Atanassov and Gargov [6] generalized the concept of the IFS to propose the interval-valued intuitionistic fuzzy set (IVIFS). The membership degree, nonmembership degree, and hesitancy degree of the IVIFS take the form of the interval values, which have more advantages in expressing the decision-makers' preference information.
For multiattribute decision-making problems with IVIF information, an important topic is the aggregation of IVIF information. Based on the algebraic operational laws on the IVIFS, Yu [7] defined the generalized interval-valued intuitionistic fuzzy weighted averaging operator and the generalized interval-valued intuitionistic fuzzy weighted geometric operator. Zhao et al. [8] proposed the generalized intervalvalued intuitionistic fuzzy ordered weighted averaging operator and the generalized interval-valued intuitionistic fuzzy hybrid averaging operator. Lin and Zhang [9] proposed some interval-valued intuitionistic fuzzy continuous operators. Wei [10] developed the induced interval-valued intuitionistic fuzzy weighted geometric operator and the induced intervalvalued intuitionistic fuzzy ordered weighted geometric operators. Yu et al. [11] developed the interval-valued intuitionistic fuzzy prioritized weighted averaging operator and the interval-valued intuitionistic fuzzy prioritized weighted geometric operator. $\mathrm{Wu}$ and $\mathrm{Su}$ [12] proposed the intervalvalued prioritized hybrid weighted operator. Zhou and $\mathrm{He}$ [13] proposed the interval-valued intuitionistic fuzzy precise 
weighted operator. Zhao and $\mathrm{Xu}$ [14] presented some new synthesized interval-valued intuitionistic fuzzy aggregation operators. Xu and Gou [15] made an overview of intervalvalued intuitionistic fuzzy aggregation operator. The operators above are based on the algebraic operational laws (i.e., the algebraic product and the algebraic sum) of the IVIFS. However, the algebraic product and the algebraic sum are not the only operational laws. The Einstein operational laws are good alternatives for information aggregation [16]. Wang and Liu [17] extended the Einstein operational laws to accommodate the environment where the aggregated information is interval-valued intuitionistic fuzzy number and proposed the interval-valued intuitionistic fuzzy Einstein weighted averaging operator, the interval-valued intuitionistic fuzzy Einstein ordered weighted averaging operator, and the interval-valued intuitionistic fuzzy Einstein hybrid weighted operator. Wang and Liu [18] proposed the interval-valued intuitionistic fuzzy Einstein weighted geometric operator, the interval-valued intuitionistic fuzzy Einstein ordered weighted geometric operator, and the interval-valued intuitionistic fuzzy Einstein hybrid weighted geometric operator. Yang and Yuan [19] presented the induced interval-valued intuitionistic fuzzy Einstein ordered weighted geometric operator. Cai and Han [20] developed the induced interval-valued Einstein ordered weighted averaging operator. The operators proposed above are based on the premise that all of the attributes are independent. However, in many real decision-making problems, the attributes are correlative or interdependent. For example, Grabisch [21] and Torra [22] gave the following classical example: "We are to evaluate a set of students in relation to three subjects: $\{$ mathematics, physics, literature\}, we want to give more importance to science-related subjects than to literature, but on the other hand we want to give some advantage to students that are good both in literature and in any of the science-related subjects." In order to solve the problem where the attributes are correlative or interactive, Choquet [23] developed the Choquet integral. Based on the Choquet integral and interval-valued intuitionistic fuzzy set, $\mathrm{Xu}$ [24] proposed the interval-valued intuitionistic fuzzy correlative averaging operator and the interval-valued intuitionistic fuzzy correlative geometric operator. Tan $[25,26]$ proposed the interval-valued intuitionistic fuzzy geometric Choquet integral operator and the interval-valued intuitionistic fuzzy Choquet integral operator. $\mathrm{Xu}$ and $\mathrm{Xia}$ [27] presented the induced generalized interval-valued intuitionistic fuzzy correlative averaging operator. Meng et al. [28] developed the generalized Banzhaf interval-valued intuitionistic fuzzy geometric Choquet integral operator. Cheng and Tang [29] proposed the interval-valued intuitionistic fuzzy generalized Shapley geometric Choquet integral operator. Gu et al. [30] proposed the interval-valued intuitionistic fuzzy Einstein correlative averaging operator. Liu et al. [31-41] proposed some Heronian mean aggregation operators and Bonferroni mean operators to solve decision-making problems. Liu et al. [42] developed the IVIFOWCS operator which combines the interval-valued intuitionistic fuzzy cosine similarity measure with the generalized ordered weighted averaging operator and applied it in the investment decision-making. Krishankumar et al. [43] developed a scientific decision framework with interval-valued intuitionistic fuzzy operator and applied it in supplier selection.

In the above studies, most interval-valued intuitionistic fuzzy Einstein operators are still used to solve the problems where the attributes are independent. The interval-valued intuitionistic fuzzy Einstein operators are seldom applied to deal with the problems where the attributes are correlative. In this paper, we developed the interval-valued intuitionistic fuzzy Einstein geometric Choquet integral operator and applied it in multiattribute group decision-making problems with interval-valued intuitionistic fuzzy information, where the attributes have interdependent phenomena. To do that, this paper is organized as follows. In Section 2, the basic knowledge of the IFS and IVIFS, Einstein operational laws of the IVIFS, and fuzzy measure are introduced. Section 3 proposes the interval-valued intuitionistic fuzzy Einstein geometric Choquet integral operator and investigates the fundamental properties of the operator. Section 4 develops a multiattribute group decision-making method based on the operator. An illustrative example is used to verify the effectiveness of the method in Section 5. The comparison with other methods is discussed in Section 6. Section 7 concludes the paper.

\section{Preliminaries}

In this section, some basic knowledge is introduced including the definitions of the intuitionistic fuzzy set (IFS), the interval-valued intuitionistic fuzzy set (IVIFS), the Einstein operational laws of IVIFS, and fuzzy measure.

Definition 1 (see [1]). Let $X$ be a universal set; an IFS $A$ in $X$ is given by

$$
A=\left\{\left(x, \mu_{A}(x), v_{A}(x)\right) \mid x \in X\right\},
$$

where $\mu_{A}(x)$ represents the membership degree, $v_{A}(x)$ represents the nonmembership degree, and $\pi_{A}=1-\mu_{A}-\nu_{A}$ is called the hesitancy degree.

Atanassov and Gargov [6] extended the IFS to propose the IVIFS; the definition of the IVIFS is given as follows.

Definition 2 (see [6]). Let $X$ be a universal set; an IVIFS $\widetilde{A}$ in $X$ is given by

$$
\begin{aligned}
& \widetilde{A}=\left\{\left(x,\left[\widetilde{\mu}_{\widetilde{A}}^{L}(x), \widetilde{\mu}_{\widetilde{A}}^{U}(x)\right],\left[\widetilde{v}^{L} \widetilde{A}(x), \widetilde{v}_{\widetilde{A}}^{U}(x)\right]\right) \mid x\right. \\
& \in X\}
\end{aligned}
$$

where the intervals $\left[\widetilde{\mu}_{\widetilde{A}^{\prime}}^{L} \widetilde{\mu}_{\widetilde{A}^{U}}^{U}\right],\left[\tilde{\nu}^{L} \widetilde{A}^{,} \widetilde{\nu}_{\widetilde{A}}^{U}\right] \subset[0,1]$ are, respectively, called the degree of membership and degree of

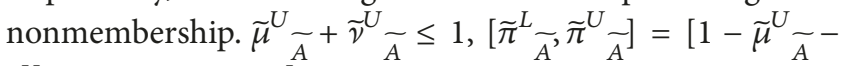
$\left.\widetilde{\nu}_{\widetilde{A}}^{U}, 1-1-\widetilde{\mu}^{L} \widetilde{A}^{-}-\widetilde{\nu}^{L}{ }_{A}\right]$ is called the degree of hesitancy. 
Presently, most proposed operators are based on the algebraic product and the algebraic sum of the IVIFS, while the algebraic product and sum are not the unique operational laws chosen to model the intersection and union on the IVIFS. Einstein product and sum are also used in intervalvalued intuitionistic fuzzy information aggregation [17].

Let $\widetilde{\alpha}_{1}=\left(\left[a_{1}, b_{1}\right],\left[c_{1}, d_{1}\right]\right)$ and $\widetilde{\alpha}_{2}=\left(\left[a_{2}, b_{2}\right],\left[c_{2}, d_{2}\right]\right)$ be two IVIFNs; some operations of Einstein product and sum on the IVIFS are given as follows:

$$
\begin{aligned}
& \tilde{a}_{1} \oplus^{\varepsilon} \widetilde{a}_{2}=\left(\left[\frac{a_{1}+a_{2}}{1+a_{1} a_{2}}, \frac{b_{1}+b_{2}}{1+b_{1} b_{2}}\right],\right. \\
& \left.\left[\frac{c_{1} c_{2}}{1+\left(1-c_{1}\right)\left(1-c_{2}\right)}, \frac{d_{1} d_{2}}{1+\left(1-d_{1}\right)\left(1-d_{2}\right)}\right]\right), \\
& \widetilde{\alpha}_{1} \otimes^{\varepsilon} \widetilde{\alpha}_{2} \\
& =\left(\left[\frac{a_{1} a_{2}}{1+\left(1-a_{1}\right)\left(1-a_{2}\right)}, \frac{b_{1} b_{2}}{1+\left(1-b_{1}\right)\left(1-b_{2}\right)}\right],\right. \\
& \left.\left[\frac{c_{1}+c_{2}}{1+c_{1} c_{2}}, \frac{d_{1}+d_{2}}{1+d_{1} d_{2}}\right]\right) \\
& \lambda \cdot{ }^{\varepsilon} \widetilde{\alpha}_{1} \\
& =\left(\left[\frac{\left(1+a_{1}\right)^{\lambda}-\left(1+a_{1}\right)^{\lambda}}{\left(1+a_{1}\right)^{\lambda}+\left(1+a_{1}\right)^{\lambda}}, \frac{\left(1+b_{1}\right)^{\lambda}-\left(1+b_{1}\right)^{\lambda}}{\left(1+b_{1}\right)^{\lambda}+\left(1+b_{1}\right)^{\lambda}}\right],\right. \\
& \left.\left[\frac{2 c_{1}^{\lambda}}{\left(2-c_{1}\right)^{\lambda}+c_{1}{ }^{\lambda}}, \frac{2 d_{1}^{\lambda}}{\left(2-d_{1}\right)^{\lambda}+d_{1}^{\lambda}}\right]\right), \quad \lambda>0, \\
& \widetilde{\alpha}_{1}^{\wedge_{\varepsilon} \lambda}=\left(\left[\frac{2 a_{1}^{\lambda}}{\left(2-a_{1}\right)^{\lambda}+a_{1}{ }^{\lambda}}, \frac{2 b_{1}^{\lambda}}{\left(2-b_{1}\right)^{\lambda}+b_{1}^{\lambda}}\right],\right. \\
& \left.\left[\frac{\left(1+c_{1}\right)^{\lambda}-\left(1-c_{1}\right)^{\lambda}}{\left(1+c_{1}\right)^{\lambda}+\left(1-c_{1}\right)^{\lambda}}, \frac{\left(1+d_{1}\right)^{\lambda}-\left(1-d_{1}\right)^{\lambda}}{\left(1+d_{1}\right)^{\lambda}+\left(1-d_{1}\right)^{\lambda}}\right]\right),
\end{aligned}
$$$$
\lambda>0 .
$$

To compare two IVIFNs, $\mathrm{Xu}$ [24] defined the score function $S(\widetilde{\alpha})=(a-c+b-d) / 2$ and accuracy function $H(\widetilde{\alpha})=(a+b+c+d) / 2$; then

(1) if $S\left(\widetilde{\alpha}_{1}\right)<S\left(\widetilde{\alpha}_{2}\right)$, then $\widetilde{\alpha}_{1}<\widetilde{\alpha}_{2}$;

(2) if $S\left(\widetilde{\alpha}_{1}\right)=S\left(\widetilde{\alpha}_{2}\right)$, then

$$
\begin{aligned}
& \text { (1) if } H\left(\widetilde{\alpha}_{1}\right)<H\left(\widetilde{\alpha}_{2}\right) \text {, then } \widetilde{\alpha}_{1}<\widetilde{\alpha}_{2} \text {; } \\
& \text { (2) if } H\left(\widetilde{\alpha}_{1}\right)>H\left(\widetilde{\alpha}_{2}\right) \text {, then } \widetilde{\alpha}_{1}>\widetilde{\alpha}_{2} \text {; } \\
& \text { (3) if } H\left(\widetilde{\alpha}_{1}\right)>H\left(\widetilde{\alpha}_{2}\right) \text {, then } \widetilde{\alpha}_{1}=\widetilde{\alpha}_{2} .
\end{aligned}
$$

Wang and Liu [18] proposed the interval-valued intuitionistic fuzzy Einstein weighted geometric $\left(\mathrm{IVIFWG}^{\varepsilon}\right.$ ) operator based on the Einstein operational laws of the IVIFS.
Definition 3 (see [18]). Let $\widetilde{\alpha}_{j}=\left(\left[a_{j}, b_{j}\right],\left[c_{j}, d_{j}\right]\right),(j=$ $1,2, \ldots, n)$, be the IVIFS. An IVIFWG ${ }^{\varepsilon}$ operator of dimension $n$ is a mapping IVIFWG ${ }^{\varepsilon}: \Omega^{n} \rightarrow \Omega$ which has a weighting vector $W$ with $w_{j} \in[0,1]$ and $\sum_{j=1}^{n} w_{j}=1$, according to the following formula:

$$
\begin{gathered}
\operatorname{IVIFWG}^{\varepsilon}=\left(\left[\frac{2 \prod_{j=1}^{n} a_{j}^{w_{j}}}{\prod_{j=1}^{n}\left(2-a_{j}\right)^{w_{j}}+\prod_{j=1}^{n} a_{j}^{w_{j}}},\right.\right. \\
\left.\frac{2 \prod_{j=1}^{n} a_{j}^{w_{j}}}{\prod_{j=1}^{n}\left(2-a_{j}\right)^{w_{j}}+\prod_{j=1}^{n} a_{j}^{w_{j}}}\right], \\
{\left[\frac{\prod_{j=1}^{n}\left(1+c_{j}\right)^{w_{j}}-\prod_{j=1}^{n}\left(1-c_{j}\right)^{w_{j}}}{\prod_{j=1}^{n}\left(1+c_{j}\right)^{w_{j}}+\prod_{j=1}^{n}\left(1-c_{j}\right)^{w_{j}}},\right.} \\
\left.\left.\frac{\prod_{j=1}^{n}\left(1+d_{j}\right)^{w_{j}}-\prod_{j=1}^{n}\left(1-c_{j}\right)^{w_{j}}}{\prod_{j=1}^{n}\left(1+c_{j}\right)^{w_{j}}+\prod_{j=1}^{n}\left(1-c_{j}\right)^{w_{j}}}\right]\right) .
\end{gathered}
$$

In real decision-making problems, the attributes of the alternatives are often interdependent or interactive. In order to solve these problems, Sugeno [44] introduced the concept of the fuzzy measure to model interaction phenomenon among combinations.

Definition 4 (see [44]). Let $S$ be a universe of discourse and let $P(S)$ be the power set of $S$. A fuzzy measure on $S$ is a set function $\mu: P(S) \rightarrow[0,1]$, satisfying the following conditions:

(1) $\mu(\phi)=0, \mu(S)=1$.

(2) $A, B \in P(S), A \subseteq B \Rightarrow \mu(A) \leq \mu(B)$.

Since the fuzzy measure is defined on the power set, it is not easy to get the fuzzy measure of each combination in a set when it is large. To increase the practicability of the fuzzy measure, Sugeno [44] introduced a special kind of fuzzy measure named the $\lambda$-fuzzy measure $g$, which is expressed by the following form:

$$
g(C \cup D)=g(C)+g(D)+\lambda g(C) g(D),
$$

where $\lambda \in(-1,+\infty)$ and $C, D \subseteq S$, with $C \cap D=\Phi$.

It is apparent that when $\lambda=0$, then $g$ is an additive measure, which means that there is no interaction between subsets $C$ and $D$. If $\lambda>0$, then $g(C \cup D)>g(C)+g(D)$, which means that $g$ is a superadditive measure and there exists complementary interaction between subsets $C$ and $D$. If $-1<\lambda<0$, then $g(C \cup D)<g(C)+g(D)$, which implies that $g$ is a subadditive measure and there exists redundancy interaction between subsets $C$ and $D$.

For finite set $S$, the $\lambda$-fuzzy measure $g$ can be equivalently expressed by 


$$
g(D)= \begin{cases}\frac{1}{\lambda}\left(\prod_{i \in D}[1+\lambda g(i)]-1\right) & \lambda \neq 0 \\ \sum_{i \in D} g(i) & \lambda=0 .\end{cases}
$$

From $\mu(S)=1$, we know that $\lambda$ is determined by $\prod_{i=1}^{n}[1+$ $\lambda g(i)]-1=\lambda$. When each $g(i)$ is given, the value of $\lambda$ can be derived from (6). For the set $S$ with $n$ elements, we only need $n$ values to get the fuzzy measure of each subset in $S$. Furthermore, if $\sum_{i=1}^{n} g(i)=1$, then $\lambda=1$.

Definition 5 (see [20]). Let $X=\left\{x_{1}, x_{2}, \ldots, x_{n}\right\}$ be a universe of discourse. Let $f$ be a positive real-valued function on $X$, and let $\mu$ be the fuzzy measure on $S$. The discrete Choquet integral of $f$ with respect to $\mu$ is defined by

$$
\begin{aligned}
C_{\mu}\left(f\left(x_{(1)}\right), f\left(x_{(2)}\right), \ldots, f\left(x_{(n)}\right)\right) \\
=\sum_{i=1}^{n}\left(\mu\left(A_{(i)}\right)-\mu\left(A_{(i+1)}\right)\right) f\left(x_{(i)}\right),
\end{aligned}
$$

where $\{(1),(2), \ldots,(n)\}$ is a permutation of $\{1,2, \ldots, n\}$, satisfying $f\left(x_{(1)}\right) \leq f\left(x_{(2)}\right) \leq \cdots f\left(x_{(n)}\right)$. And $A_{(i)}=$ $\left\{x_{(i)}, x_{(i+1)}, \ldots, x_{(n)}\right\} ; A_{(n+1)}=\phi$.

\section{Interval-Valued Intuitionistic Fuzzy Einstein Geometric Choquet Integral Operator}

In many decision-making problems, the criteria of alternatives often are correlative or interdependent. To solve these problems, the interval-valued intuitionistic fuzzy Einstein geometric Choquet integral operator is proposed and the properties of the operator are investigated as follows.

Definition 6. Let $\widetilde{\alpha}_{j}=\left(\left[a_{j}, b_{j}\right],\left[c_{j}, d_{j}\right]\right),(j=1,2, \ldots, n)$, be an IVIFS on $X=\left(x_{1}, x_{2}, \ldots, x_{n}\right)$; let $\mu$ be the fuzzy measure on $X$. An interval-valued intuitionistic fuzzy Einstein geometric Choquet integral operator of dimension $n$ is a mapping IVIFEGC: $\Omega^{n} \rightarrow \Omega$ such that

$$
\begin{aligned}
& \operatorname{IVIFEGC}\left(\widetilde{\alpha}_{1}, \widetilde{\alpha}_{2}, \ldots, \widetilde{\alpha}_{n}\right) \\
& =\bigoplus^{\varepsilon} \widetilde{\alpha}_{\sigma(j)}\left(g\left(A_{(j)}\right)-g\left(A_{(j+1)}\right)\right),
\end{aligned}
$$

where $\{\sigma(1), \sigma(2), \ldots, \sigma(n)\}$ is a permutation of $\{1,2, \ldots, n\}$, satisfying $\tilde{a}_{\sigma(1)} \leq \tilde{a}_{\sigma(2)} \leq \cdots \leq \tilde{a}_{\sigma(n)}$ and $A_{(j)}=$ $\left\{x_{(j)}, x_{(j+1)}, \ldots, x_{(n)}\right\}$ and $A_{(n+1)}=\phi$.

The IVIFEGC operator can also be represented by the following equation:

$$
\begin{gathered}
\text { IVIFEGC }=\left[\frac{2 \prod_{j=1}^{n} a_{\sigma(j)} w_{\sigma(j)}}{\prod_{j=1}^{n}\left(2-a_{\sigma(j)}\right)^{w_{\sigma(j)}}+\prod_{j=1}^{n} a_{\sigma(j)} w_{\sigma(j)}},\right. \\
\left.\frac{2 \prod_{j=1}^{n} b_{\sigma(j)} w_{\sigma(j)}}{\left.\prod_{j=1}^{n}\left(2-b_{\sigma(j)}\right)\right)^{w_{\sigma(j)}}+\prod_{j=1}^{n} b_{\sigma(j)} w_{\sigma(j)}}\right], \\
{\left[\frac{\prod_{j=1}^{n}\left(1+c_{\sigma(j)}\right)^{w_{\sigma(j)}}-\prod_{j=1}^{n}\left(1-c_{\sigma(j)}\right)^{w_{\sigma(j)}}}{\prod_{j=1}^{n}\left(1+c_{\sigma(j)}\right)^{w_{\sigma(j)}}+\prod_{j=1}^{n}\left(1-c_{\sigma(j)}\right)^{w_{\sigma(j)}}},\right.} \\
\left.\frac{\prod_{j=1}^{n}\left(1+d_{\sigma(j)}\right)^{w_{\sigma(j)}}-\prod_{j=1}^{n}\left(1-d_{\sigma(j)}\right)^{w_{\sigma(j)}}}{\prod_{j=1}^{n}\left(1+d_{\sigma(j)}\right)^{w_{\sigma(j)}}+\prod_{j=1}^{n}\left(1-d_{\sigma(j)}\right)^{w_{\sigma(j)}}}\right]
\end{gathered}
$$
gated.

Next the properties of the operator are further investi-

Theorem 7 (idempotency). Let $\tilde{\alpha}_{j}=\left(\left[a_{j}, b_{j}\right],\left[c_{j}, d_{j}\right]\right), \quad(j=$ $1,2, \ldots, n)$, be an IVIFS on $X=\left(x_{1}, x_{2}, \ldots, x_{n}\right)$, and let $\mu$ be a fuzzy measure on X. If all IVIFNs are equal (i.e., $\tilde{\alpha}_{j}=\alpha=$ $([a, b],[c, d]))$, then

$$
\operatorname{IVIFEGC}\left(\widetilde{\alpha}_{1}, \widetilde{\alpha}_{2}, \ldots, \widetilde{\alpha}_{n}\right)=\widetilde{\alpha} .
$$

Proof. According to Theorem 7, if all IVIFNs are equal, then

$$
\operatorname{IVIFEGC~}=\left(\begin{array}{c}
{\left[\frac{2 a^{\sum_{j=1}^{n} w_{\sigma(j)}}}{(2-a)^{\sum_{j=1}^{n} w_{\sigma(j)}}+a^{\sum_{j=1}^{n} w_{\sigma(j)}}}, \frac{2 b^{\sum_{j=1}^{n} w_{\sigma(j)}}}{(2-b)^{\sum_{j=1}^{n} w_{\sigma(j)}}+b^{\sum_{j=1}^{n} w_{\sigma(j)}}}\right]} \\
{\left[\frac{(1+c)^{\sum_{j=1}^{n} w_{\sigma(j)}}-(1-c)^{\sum_{j=1}^{n} w_{\sigma(j)}}}{(1+c)^{\sum_{j=1}^{n} w_{\sigma(j)}}+(1-c)^{\sum_{j=1}^{n} w_{\sigma(j)}}}, \frac{(1+d)^{\sum_{j=1}^{n} w_{\sigma(j)}}-(1-d)^{\sum_{j=1}^{n} w_{\sigma(j)}}}{(1+d)^{\sum_{j=1}^{n} w_{\sigma(j)}}+(1-d)^{\sum_{j=1}^{n} w_{\sigma(j)}}}\right.}
\end{array}\right) .
$$

Since

$$
\begin{aligned}
\sum_{j=1}^{n} w_{\sigma(j)} & =\sum_{j=1}^{n} \mu\left(\left(A_{(j)}\right)-\mu\left(A_{(j+1)}\right)\right) \\
& =\mu\left(A_{(1)}\right)-\mu\left(A_{(n+1)}\right)=1,
\end{aligned}
$$

then

$$
\operatorname{IVIFEGC}\left(\widetilde{\alpha}_{1}, \widetilde{\alpha}_{2}, \ldots, \widetilde{\alpha}_{n}\right)=\widetilde{\alpha}
$$

Theorem 8 (monotonicity). Let $\widetilde{\alpha}_{j}=\left(\left[a_{j}, b_{j}\right],\left[c_{j}, d_{j}\right]\right), \widetilde{\beta}_{j}=$ $\left(\left[a^{\prime}{ }_{j}, b^{\prime}{ }_{j}\right],\left[c^{\prime}{ }_{j}, d^{\prime}{ }_{j}\right]\right),(j=1,2, \ldots, n)$, be two IVIFSs on $X=$ $\left(x_{1}, x_{2}, \ldots, x_{n}\right)$, let $\mu$ be a fuzzy measure on $X$, and let $(j)$ be a permutation on $X$ such that $\widetilde{\alpha}_{\sigma(j)} \leq \widetilde{\alpha}_{(j+1),}, \widetilde{\beta}_{\sigma(j)} \leq \widetilde{\beta}_{(j+1)}$; if $a_{\sigma(j)} \leq a_{(j)}^{\prime}, b_{\sigma(j)} \leq b_{(j)}^{\prime}, c_{\sigma(j)} \geq c_{(j)}^{\prime}, d_{\sigma(j)} \geq d_{(j)}^{\prime}$, then

$$
\begin{aligned}
& \operatorname{IVIFEGC}\left(\widetilde{\alpha}_{1}, \widetilde{\alpha}_{2}, \ldots, \widetilde{\alpha}_{n}\right) \\
& \quad \leq \operatorname{IVIFEGC}\left(\widetilde{\beta}_{1}, \widetilde{\beta}_{2}, \ldots, \widetilde{\beta}_{n}\right) .
\end{aligned}
$$


Proof. Since $A_{(n+1)} \subseteq A_{(n)}$, then $\mu\left(A_{(j)}\right)-\mu\left(A_{(j+1)}\right) \geq 0$.

For any $j, a_{\sigma(j)} \leq a_{(j)}^{\prime}$ and $b_{\sigma(j)} \leq b_{(j)}^{\prime}$; then

$$
\begin{aligned}
& \prod_{j=1}^{n}\left(\frac{2-a_{\sigma(j)}}{a_{\sigma(j)}}\right)^{w_{\sigma(j)}} \geq \prod_{j=1}^{n}\left(\frac{2-a_{\sigma(j)}^{\prime}}{a_{\sigma(j)}^{\prime}}\right)^{w_{\sigma(j)}} \Longleftrightarrow, \\
& \frac{2}{\prod_{j=1}^{n}\left(\left(2-a_{\sigma(j)}^{\prime}\right) / a_{\sigma(j)}^{\prime}\right)^{w_{\sigma(j)}}+1} \\
& \geq \frac{2}{\prod_{j=1}^{n}\left(\left(2-a_{\sigma(j)}\right) / a_{\sigma(j)}\right)^{w_{\sigma(j)}}+1} ;
\end{aligned}
$$

that is,

$$
\begin{gathered}
\frac{2 \prod_{j=1}^{n}\left(a_{\sigma(j)}^{\prime}\right)^{w_{\sigma(j)}}}{\prod_{j=1}^{n}\left(2-a_{\sigma(j)}^{\prime}\right)^{w_{\sigma(j)}}+\prod_{j=1}^{n}\left(a_{\sigma(j)}^{\prime}\right)^{w_{\sigma(j)}}} \\
\geq \frac{2 \prod_{j=1}^{n}\left(a_{\sigma(j)}\right)^{w_{\sigma(j)}}}{\prod_{j=1}^{n}\left(2-a_{\sigma(j)}\right)^{w_{\sigma(j)}}+\prod_{j=1}^{n}\left(a_{\sigma(j)}\right)^{w_{\sigma(j)}}} .
\end{gathered}
$$

Also,

$$
\begin{gathered}
\frac{2 \prod_{j=1}^{n}\left(b_{\sigma(j)}\right)^{w_{\sigma(j)}}}{\prod_{j=1}^{n}\left(2-b_{\sigma(j)}\right)^{w_{\sigma(j)}}+\prod_{j=1}^{n}\left(b_{\sigma(j)}\right)^{w_{\sigma(j)}}} \\
\geq \frac{2 \prod_{j=1}^{n}\left(b_{\sigma(j)}^{\prime}\right)^{w_{\sigma(j)}}}{\prod_{j=1}^{n}\left(2-b_{\sigma(j)}^{\prime}\right)^{w_{\sigma(j)}}+\prod_{j=1}^{n}\left(b_{\sigma(j)}^{\prime}\right)^{w_{\sigma(j)}}} .
\end{gathered}
$$

For any $j, c_{\sigma(j)} \geq c_{\sigma(j)}^{\prime}, d_{\sigma(j)} \geq d_{\sigma(j)}^{\prime}$; then

$$
\begin{gathered}
\prod_{j=1}^{n}\left(\frac{1-c_{\sigma(j)}}{1+c_{\sigma(j)}}\right)^{w_{\sigma(j)}} \leq \prod_{j=1}^{n}\left(\frac{1-c_{\sigma(j)}^{\prime}}{1+c_{\sigma(j)}^{\prime}}\right)^{w_{\sigma(j)}} \Longleftrightarrow, \\
\frac{2}{1+\prod_{j=1}^{n}\left(\left(1-c_{\sigma(j)}\right) /\left(1+c_{\sigma(j)}\right)\right)^{w_{\sigma(j)}}-1} \\
\geq \frac{2}{1+\prod_{j=1}^{n}\left(\left(1-c_{\sigma(j)}^{\prime}\right) /\left(1+c_{\sigma(j)}^{\prime}\right)\right)^{w_{\sigma(j)}}}-1 ;
\end{gathered}
$$

that is,

$$
\begin{aligned}
& \frac{\prod_{j=1}^{n}\left(1+c_{\sigma(j)}\right)^{w_{\sigma(j)}}-\prod_{j=1}^{n}\left(1-c_{\sigma(j)}\right)^{w_{\sigma(j)}}}{\prod_{j=1}^{n}\left(1+c_{\sigma(j)}\right)^{w_{\sigma(j)}}+\prod_{j=1}^{n}\left(1-c_{\sigma(j)}\right)^{w_{\sigma(j)}}} \\
& \quad \geq \frac{\prod_{j=1}^{n}\left(1+c_{\sigma(j)}^{\prime}\right)^{w_{\sigma(j)}}-\prod_{j=1}^{n}\left(1-c_{\sigma(j)}^{\prime}\right)^{w_{\sigma(j)}}}{\prod_{j=1}^{n}\left(1+c_{\sigma(j)}^{\prime}\right)^{w_{\sigma(j)}}+\prod_{j=1}^{n}\left(1-c_{\sigma(j)}^{\prime}\right)^{w_{\sigma(j)}}} .
\end{aligned}
$$

According to the score function and accuracy function, we have

$$
\begin{aligned}
& \operatorname{IVIFEGC}\left(\widetilde{\alpha}_{1}, \widetilde{\alpha}_{2}, \ldots, \widetilde{\alpha}_{n}\right) \\
& \quad \leq \operatorname{IVIFEGC}\left(\widetilde{\beta}_{1}, \widetilde{\beta}_{2}, \ldots, \widetilde{\beta}_{n}\right) .
\end{aligned}
$$

Theorem 9 (boundedness). Let $\widetilde{\alpha}_{j}=\left(\left[a_{j}, b_{j}\right],\left[c_{j}, d_{j}\right]\right),(j=$ $1,2, \ldots, n)$, be an IVIFS on $X=\left(x_{1}, x_{2}, \ldots, x_{n}\right)$, and let $\mu$ be a fuzzy measure on $X$; if

$$
\begin{aligned}
& \widetilde{\alpha}^{-}=\left(\left[\min _{j} a_{j}, \min _{j} b_{j}\right],\left[\max _{j} c_{j}, \max _{j} d_{j}\right]\right), \\
& \tilde{\alpha}^{+}=\left(\left[\max _{j} a_{j}, \max _{j} b_{j}\right],\left[\min _{j} c_{j}, \min _{j} d_{j}\right]\right),
\end{aligned}
$$

then

$$
\widetilde{\alpha}^{-} \leq \operatorname{IVIFEGC}\left(\widetilde{\alpha}_{1}, \widetilde{\alpha}_{2}, \ldots, \widetilde{\alpha}_{n}\right) \leq \widetilde{\alpha}^{+} .
$$

Proof. For all $\widetilde{\alpha}_{j}=\left(\left[a_{j}, b_{j}\right],\left[c_{j}, d_{j}\right]\right), \widetilde{\alpha}^{-}$and $\widetilde{\alpha}^{+}$are IVIFNs. Since $A_{(i+1)} \subseteq A_{(i)}$, then $\mu\left(A_{(j)}\right)-\mu\left(A_{(j+1)}\right) \geq 0$.

Since

$$
\begin{aligned}
& \min _{j} a_{j} \leq a_{\sigma(j)} \leq \max _{j} a_{j}, \\
& \min _{j} b_{j} \leq b_{\sigma(j)} \leq \max _{j} b_{j}, \\
& \min _{j} c_{j} \leq c_{\sigma(j)} \leq \max _{j} c_{j}, \\
& \min _{j} d_{j} \leq d_{\sigma(j)} \leq \max _{j} d_{j} ;
\end{aligned}
$$

then

$$
\begin{aligned}
& \frac{2 \prod_{j=1}^{n}\left(\min _{j} a_{j}\right)^{w_{\sigma(j)}}}{\prod_{j=1}^{n}\left(2-\min _{j} b_{j}\right)^{w_{\sigma(j)}}+\prod_{j=1}^{n}\left(\min _{j} b_{j}\right)^{w_{\sigma(j)}}} \\
& \leq \frac{2 \prod_{j=1}^{n}\left(b_{\sigma(j)}\right)^{w_{\sigma(j)}}}{\prod_{j=1}^{n}\left(2-b_{\sigma(j)}\right)^{w_{\sigma(j)}}+\prod_{j=1}^{n}\left(b_{\sigma(j)}\right)^{w_{\sigma(j)}}} \\
& \leq \frac{2 \prod_{j=1}^{n}\left(\max _{j} b_{j}\right)^{w_{\sigma(j)}}}{\prod_{j=1}^{n}\left(2-\max _{j} b_{j}\right)^{w_{\sigma(j)}}+\prod_{j=1}^{n}\left(\max _{j} b_{j}\right)^{w_{\sigma(j)}}}, \\
& \frac{2 \prod_{j=1}^{n}\left(\min _{j} b_{j}\right)^{w_{\sigma(j)}}}{\prod_{j=1}^{n}\left(2-\min _{j} b_{j}\right)^{w_{\sigma(j)}}+\prod_{j=1}^{n}\left(\min _{j} b_{j}\right)^{w_{\sigma(j)}}} \\
& \leq \frac{2 \prod_{j=1}^{n}\left(b_{\sigma(j)}\right)^{w_{\sigma(j)}}}{\prod_{j=1}^{n}\left(2-b_{\sigma(j)}\right)^{w_{\sigma(j)}}+\prod_{j=1}^{n}\left(b_{\sigma(j)}\right)^{w_{\sigma(j)}}} \\
& \leq \frac{2 \prod_{j=1}^{n}\left(\max _{j} b_{j}\right)^{w_{\sigma(j)}}}{\prod_{j=1}^{n}\left(2-\max _{j} b_{j}\right)^{w_{\sigma(j)}}+\prod_{j=1}^{n}\left(\max _{j} b_{j}\right)^{w_{\sigma(j)}}}, \\
& \frac{\prod_{j=1}^{n}\left(1+\min _{j} c_{j}\right)^{w_{\sigma(j)}}-\prod_{j=1}^{n}\left(1-\min _{j} c_{j}\right)^{w_{\sigma(j)}}}{\prod_{j=1}^{n}\left(1+\min _{j} c_{j}\right)^{w_{\sigma(j)}}+\prod_{j=1}^{n}\left(1-\min _{j} c_{j}\right)^{w_{\sigma(j)}}}
\end{aligned}
$$




$$
\begin{aligned}
& \leq \frac{\prod_{j=1}^{n}\left(1+c_{\sigma(j)}\right)^{w_{\sigma(j)}}-\prod_{j=1}^{n}\left(1-c_{\sigma(j)}\right)^{w_{\sigma(j)}}}{\prod_{j=1}^{n}\left(1+c_{\sigma(j)}\right)^{w_{\sigma(j)}}+\prod_{j=1}^{n}\left(1-c_{\sigma(j)}\right)^{w_{\sigma(j)}}} \\
& \leq \frac{\prod_{j=1}^{n}\left(1+\max _{j} c_{j}\right)^{w_{\sigma(j)}}-\prod_{j=1}^{n}\left(1-\max _{j} c_{j}\right)^{w_{\sigma(j)}}}{\prod_{j=1}^{n}\left(1+\max _{j} c_{j}\right)^{w_{\sigma(j)}}+\prod_{j=1}^{n}\left(1-\max _{j} c_{j}\right)^{w_{\sigma(j)}}}, \\
& \frac{\prod_{j=1}^{n}\left(1+\min _{j} d_{j}\right)^{w_{\sigma(j)}}-\prod_{j=1}^{n}\left(1-\min _{j} d_{j}\right)^{w_{\sigma(j)}}}{\prod_{j=1}^{n}\left(1+\min _{j} d_{j}\right)^{w_{\sigma(j)}}+\prod_{j=1}^{n}\left(1-\min _{j} d_{j}\right)^{w_{\sigma(j)}}} \\
& \leq \frac{\prod_{j=1}^{n}\left(1+d_{\sigma(j)}\right)^{w_{\sigma(j)}}-\prod_{j=1}^{n}\left(1-d_{\sigma(j)}\right)^{w_{\sigma(j)}}}{\prod_{j=1}^{n}\left(1+d_{\sigma(j)}\right)^{w_{\sigma(j)}}+\prod_{j=1}^{n}\left(1-d_{\sigma(j)}\right)^{w_{\sigma(j)}}} \\
& \leq \frac{\prod_{j=1}^{n}\left(1+\max _{j} d_{j}\right)^{w_{\sigma(j)}}-\prod_{j=1}^{n}\left(1-\max _{j} d_{j}\right)^{w_{\sigma(j)}}}{\prod_{j=1}^{n}\left(1+\max _{j} d_{j}\right)^{w_{\sigma(j)}}+\prod_{j=1}^{n}\left(1-\max _{j} d_{j}\right)^{w_{\sigma(j)}}} .
\end{aligned}
$$

Since

$$
\begin{aligned}
\sum_{j=1}^{n} w_{\sigma(j)} & =\sum_{j=1}^{n}\left(\mu\left(A_{(j)}\right)-\mu\left(A_{(j+1)}\right)\right) \\
& =\mu\left(A_{(1)}\right)-\mu\left(A_{(n+1)}\right)=1
\end{aligned}
$$

and according to Theorem 7, we have

$$
\begin{aligned}
\min _{j} a_{j} & \leq \frac{2 \prod_{j=1}^{n}\left(a_{\sigma(j)}\right)^{w_{\sigma(j)}}}{\prod_{j=1}^{n}\left(2-a_{\sigma(j)}\right)^{w_{\sigma(j)}}+\prod_{j=1}^{n}\left(a_{\sigma(j)}\right)^{w_{\sigma(j)}}} \\
& \leq \max _{j} a_{j}, \\
\min _{j} b_{j} & \leq \frac{2 \prod_{j=1}^{n}\left(b_{\sigma(j)}\right)^{w_{\sigma(j)}}}{\prod_{j=1}^{n}\left(2-b_{\sigma(j)}\right)^{w_{\sigma(j)}}+\prod_{j=1}^{n}\left(b_{\sigma(j)}\right)^{w_{\sigma(j)}}} \\
& \leq \max _{j} b_{j}, \\
\min _{j} c_{j} & \leq \frac{\prod_{j=1}^{n}\left(1+c_{\sigma(j)}\right)^{w_{\sigma(j)}}-\prod_{j=1}^{n}\left(1-c_{\sigma(j)}\right)^{w_{\sigma(j)}}}{\prod_{j=1}^{n}\left(1+c_{\sigma(j)}\right)^{w_{\sigma(j)}}+\prod_{j=1}^{n}\left(1-c_{\sigma(j)}\right)^{w_{\sigma(j)}}} \\
& \leq \max _{j} c_{j}, \\
\min _{j} d_{j} & \leq \frac{\prod_{j=1}^{n}\left(1+d_{\sigma(j)}\right)^{w_{\sigma(j)}}-\prod_{j=1}^{n}\left(1-d_{\sigma(j)}\right)^{w_{\sigma(j)}}}{\prod_{j=1}^{n}\left(1+d_{\sigma(j)}\right)^{w_{\sigma(j)}}+\prod_{j=1}^{n}\left(1-d_{\sigma(j)}\right)^{w_{\sigma(j)}}} \\
& \leq \max _{j} d_{j},
\end{aligned}
$$

$\left(\left[\min _{j} a_{j}, \min _{j} b_{j}\right],\left[\max _{j} c_{j}, \max _{j} d_{j}\right]\right) \leq \operatorname{IVIFEGC}\left(\widetilde{\alpha}_{1}, \widetilde{\alpha}_{2}, \ldots\right.$, $\left.\widetilde{\alpha}_{n}\right) \leq\left(\left[\max _{j} a_{j}, \max _{j} b_{j}\right],\left[\min _{j} c_{j}, \min _{j} d_{j}\right]\right)$; that is,

$$
\widetilde{\alpha}^{-} \leq \operatorname{IVIFEGC}\left(\widetilde{\alpha}_{1}, \widetilde{\alpha}_{2}, \ldots, \widetilde{\alpha}_{n}\right) \leq \widetilde{\alpha}^{+} .
$$

Theorem 10 (commutativity). Let $\tilde{\alpha}_{j}=\left(\left[a_{j}, b_{j}\right],\left[c_{j}, d_{j}\right]\right)$, $(j=1,2, \ldots, n)$, be an IVIFS on $X=\left(x_{1}, x_{2}, \ldots, x_{n}\right)$, and let $\mu$ be a fuzzy measure on $X ;\left(\widetilde{\alpha}_{1}^{\prime}, \widetilde{\alpha}_{2}^{\prime}, \ldots, \widetilde{\alpha}_{n}^{\prime}\right)$ is any permutation of $\left(\widetilde{a}_{1}, \widetilde{a}_{2}, \ldots, \tilde{a}_{n}\right)$; then

$$
\begin{aligned}
& \operatorname{IVIFEGC}\left(\widetilde{\alpha}_{1}, \widetilde{\alpha}_{2}, \ldots, \widetilde{\alpha}_{n}\right) \\
& \quad=\operatorname{IVIFEGC}\left(\widetilde{\alpha}_{1}^{\prime}, \widetilde{\alpha}_{2}^{\prime}, \ldots, \widetilde{\alpha}_{n}^{\prime}\right) .
\end{aligned}
$$

Proof. According to the definition of the IVIFEGC operator, Theorem 10 can be proven.

\section{The Multiattribute Group Decision-Making Approach Based on Interval-Valued Intuitionistic Fuzzy Einstein Geometric Choquet Integral Operator}

Considering the multiattribute group decision-making problem under interval-valued intuitionistic fuzzy environment, let $E=\left\{e_{1}, e_{2}, \ldots, e_{t}\right\}$ be the set of experts, let $A=\left\{a_{1}, a_{2}, \ldots\right.$, $\left.a_{m}\right\}$ be a set of alternatives, and let $C=\left\{c_{1}, c_{2}, \ldots, c_{n}\right\}$ be a set of attributes. The decision-making matrix $A^{k}=\left(r_{i j}{ }^{(k)}\right)$ is given by expert $e_{k} \in E$, where $\left(r_{i j}{ }^{(k)}\right)=\left(\left[\mu_{i j}{ }^{(k)} \mu_{i j}{ }^{(k)}\right]\right.$, $\left.\left[v_{i j}{ }^{(k)}, v_{i j}{ }^{(k)}\right]\right)$ is the evaluation value given by expert $e_{k}$ on the alternatives $a_{i}$ with respect to the attribute $c_{j}$.

Step 1. Determine the fuzzy measure of the individual expert and subset of the experts.

By employing the experience of the experts, the fuzzy measure of the individual expert can be confirmed (i.e., $\left.g_{i}=g\left(e_{i}\right)\right)$. According to (6), the parameter $\lambda_{1}$ and the fuzzy measure of the subset of the experts can be determined.

Step 2. Utilize the IVIFEGC operator to aggregate the decision-making matrix $A^{k}=\left(r_{i j}{ }^{(k)}\right), k=1,2, \ldots, t$, into the comprehensive decision-making matrix $A=\left(r_{i j}\right)_{m \times n}$.

Step 3. Determine the fuzzy measure of the criterion and the subset of criteria.

The experts confirm the fuzzy measure of the criterion empirically (i.e., $g_{i}=g\left(c_{i}\right)$ ). By utilizing (6), the parameter $\lambda_{2}$ and the fuzzy measure of the subset of the criteria can be determined.

Step 4. Utilize the IVIFEGC operator to obtain the overall preference of the alternative $a_{i}$.

Step 5. Rank the alternatives according to the score of the alternatives, the greater the score of $S\left(a_{i}\right)$, the better the alternative $a_{i}$.

\section{An Application Example}

With the increasingly fierce competition in the market, many traditional manufacturers usually outsource their noncore businesses and focus on core business, such as production design, marketing, and after-sale service. AUX is a famous air conditioner manufacturer in China; it wants to outsource the 
TABLE 1: Evaluation matrices.

\begin{tabular}{|c|c|c|c|c|}
\hline & $a_{1}$ & $a_{2}$ & $a_{3}$ & $a_{4}$ \\
\hline \multicolumn{5}{|l|}{$e_{1}$} \\
\hline$c_{1}$ & $([0.5,0.6],[0.1,0.3])$ & $([0.6,0.7],[0.1,0.3])$ & $([0.7,0.8],[0.1,0.2])$ & $([0.4,0.5],[0.3,0.4])$ \\
\hline$c_{2}$ & $([0.6,0.7],[0.1,0.2])$ & $([0.5,0.6],[0.2,0.3])$ & $([0.5,0.6],[0.1,0.2])$ & $([0.5,0.6],[0.2,0.3])$ \\
\hline$c_{3}$ & $([0.4,0.5],[0.1,0.3])$ & $([0.6,0.7],[0.1,0.2])$ & $([0.5,0.6],[0.2,0.3])$ & $([0.5,0.6],[0.1,0.3])$ \\
\hline$c_{4}$ & $([0.5,0.6],[0.2,0.3])$ & $([0.6,0.8],[0.1,0.2])$ & $([0.5,0.7],[0.1,0.2])$ & $([0.4,0.8],[0.1,0.2])$ \\
\hline$c_{5}$ & $([0.5,0.6],[0.3,0.4])$ & $([0.4,0.5],[0.3,0.4])$ & $([0.2,0.3],[0.5,0.6])$ & $([0.5,0.6],[0.1,0.2])$ \\
\hline \multicolumn{5}{|c|}{$e_{2}$} \\
\hline$c_{1}$ & $([0.5,0.7],[0.1,0.2])$ & $([0.5,0.6],[0.1,0.2])$ & $([0.6,0.7],[0.1,0.2])$ & $([0.5,0.6],[0.2,0.3])$ \\
\hline$c_{2}$ & $([0.5,0.6],[0.2,0.3])$ & $([0.4,0.7],[0.2,0.3])$ & $([0.5,0.6],[0.1,0.2])$ & $([0.4,0.5],[0.3,0.4])$ \\
\hline$c_{3}$ & $([0.6,0.8],[0.1,0.2])$ & $([0.5,0.6],[0.2,0.3])$ & $([0.6,0.7],[0.2,0.3])$ & $([0.3,0.4],[0.4,0.5])$ \\
\hline$c_{4}$ & $([0.6,0.7],[0.2,0.3])$ & $([0.4,0.8],[0.1,0.2])$ & $([0.5,0.6],[0.2,0.3])$ & $([0.6,0.7],[0.1,0.2])$ \\
\hline$c_{5}$ & $([0.3,0.5],[0.2,0.3])$ & $([0.4,0.5],[0.3,0.4])$ & $([0.3,0.4],[0.5,0.6])$ & $([0.5,0.6],[0.1,0.2])$ \\
\hline \multicolumn{5}{|c|}{$e_{3}$} \\
\hline$c_{1}$ & $([0.5,0.6],[0.2,0.4])$ & $([0.5,0.6],[0.1,0.3])$ & $([0.6,0.8],[0.1,0.2])$ & $([0.6,0.7],[0.1,0.2])$ \\
\hline$c_{2}$ & $([0.7,0.8],[0.1,0.2])$ & $([0.7,0.8],[0.1,0.2])$ & $([0.5,0.6],[0.1,0.3])$ & $([0.5,0.6],[0.2,0.3])$ \\
\hline$c_{3}$ & $([0.6,0.7],[0.2,0.3])$ & $([0.4,0.6],[0.3,0.4])$ & $([0.5,0.6],[0.2,0.3])$ & $([0.4,0.6],[0.1,0.2])$ \\
\hline$c_{4}$ & $([0.4,0.5],[0.1,0.2])$ & $([0.6,0.7],[0.1,0.2])$ & $([0.6,0.7],[0.1,0.2])$ & $([0.6,0.7],[0.2,0.3])$ \\
\hline$c_{5}$ & $([0.4,0.5],[0.2,0.4])$ & $([0.4,0.5],[0.1,0.2])$ & $([0.2,0.3],[0.5,0.6])$ & $([0.4,0.5],[0.4,0.5])$ \\
\hline \multicolumn{5}{|c|}{$e_{4}$} \\
\hline$c_{1}$ & $([0.6,0.7],[0.1,0.2])$ & $([0.5,0.6],[0.2,0.3])$ & $([0.7,0.8],[0.1,0.2])$ & $([0.6,0.7],[0.2,0.3])$ \\
\hline$c_{2}$ & $([0.5,0.7],[0.2,0.3])$ & $([0.6,0.7],[0.1,0.2])$ & $([0.6,0.7],[0.1,0.3])$ & $([0.5,0.6],[0.1,0.2])$ \\
\hline$c_{3}$ & $([0.5,0.6],[0.1,0.2])$ & $([0.5,0.6],[0.3,0.4])$ & $([0.5,0.6],[0.1,0.2])$ & $([0.5,0.7],[0.1,0.2])$ \\
\hline$c_{4}$ & $([0.5,0.6],[0.2,0.3])$ & $([0.6,0.7],[0.1,0.2])$ & $([0.5,0.6],[0.2,0.3])$ & $([0.6,0.7],[0.2,0.3])$ \\
\hline$c_{5}$ & $([0.3,0.5],[0.3,0.4])$ & $([0.4,0.5],[0.2,0.3])$ & $([0.2,0.3],[0.6,0.7])$ & $([0.3,0.4],[0.4,0.5])$ \\
\hline \multicolumn{5}{|l|}{$e_{5}$} \\
\hline$c_{1}$ & $([0.5,0.6],[0.3,0.4])$ & $([0.6,0.7],[0.1,0.3])$ & $([0.6,0.8],[0.1,0.2])$ & $([0.5,0.6],[0.1,0.3])$ \\
\hline$c_{2}$ & $([0.6,0.7],[0.1,0.2])$ & $([0.6,0.7],[0.2,0.3])$ & $([0.5,0.6],[0.2,0.3])$ & $([0.5,0.7],[0.1,0.2])$ \\
\hline$c_{3}$ & $([0.5,0.6],[0.2,0.3])$ & $([0.5,0.6],[0.1,0.2])$ & $([0.5,0.6],[0.2,0.4])$ & $([0.5,0.6],[0.2,0.3])$ \\
\hline$c_{4}$ & $([0.5,0.7],[0.2,0.3])$ & $([0.5,0.6],[0.1,0.2])$ & $([0.6,0.7],[0.2,0.3])$ & $([0.6,0.7],[0.2,0.3])$ \\
\hline$c_{5}$ & $([0.3,0.4],[0.3,0.4])$ & $([0.4,0.5],[0.3,0.4])$ & $([0.2,0.3],[0.5,0.6])$ & $([0.2,0.3],[0.4,0.5])$ \\
\hline
\end{tabular}

logistics to reduce the logistics cost and improve the satisfaction of the customers. After the first round screening, four alternatives are selected, which are $A=\left\{a_{1}, a_{2}, a_{3}, a_{4}\right\}$.The evaluation criteria include service quality $\left(c_{1}\right)$, response ability $\left(c_{2}\right)$, flexibility $\left(c_{3}\right)$, technology $\left(c_{4}\right)$, and cost $\left(c_{5}\right)$. Obviously, these evaluation criteria are mutually interactive. For example, there exists tradeoff between service quality and cost. Response ability, flexibility, and technology still have some effects on the service quality. The manufacturer invites five experts $E=\left\{e_{1}, e_{2}, e_{3}, e_{4}, e_{5}\right\}$ to evaluate and select the appropriate logistics service provider. The alternatives are to be evaluated using the interval-valued intuitionistic fuzzy number by experts, as listed in Table 1.

Step 1. By employing the experience of the experts, the fuzzy measure of the individual expert can be confirmed; that is, $g\left(e_{1}\right)=0.15, g\left(e_{2}\right)=0.17, g\left(e_{3}\right)=0.2, g\left(e_{4}\right)=0.3$, and $g\left(e_{5}\right)=0.25$. According to (6), the parameter $\lambda_{1}=-0.16$ and the fuzzy measure of the subset of the experts can be determined as listed in Table 2.
Step 2. Utilize the IVIFEGC operator to aggregate the decision-making matrices of Table 1 into the comprehensive decision-making matrix, which is shown in Table 3.

Step 3. Determine the fuzzy measure of the criterion and the subset of criteria.

The experts confirm the fuzzy measure of the criterion empirically; that is, $g\left(c_{1}\right)=0.3, g\left(c_{2}\right)=0.25, g\left(c_{3}\right)=0.25$, $g\left(c_{4}\right)=0.3$, and $g\left(c_{5}\right)=0.35$. By utilizing (6), the parameter $\lambda_{2}=-0.65$ and the fuzzy measure of the subset of the criteria can be determined in Table 4 .

Step 4. Utilize the IVIFEGC operator to obtain the overall preference of the alternative $a_{i}$ :

$$
\begin{aligned}
& a_{1}=([0.5012,0.6255],[0.1740,0.2795]) ; \\
& a_{2}=([0.5193,0.6477],[0.1519,0.2642]) ; \\
& a_{3}=([0.5012,0.6255],[0.1740,0.2795]) ; \\
& a_{4}=([0.4894,0.6163],[0.1912,0.3108]) .
\end{aligned}
$$


TABLE 2: The fuzzy measure of the individual expert and the subset of the experts.

\begin{tabular}{lc}
\hline$c_{i}$ & $g\left(c_{i}\right)$ \\
\hline$\{1\}$ & 0.15 \\
$\{2\}$ & 0.17 \\
$\{3\}$ & 0.2 \\
$\{4\}$ & 0.3 \\
$\{5\}$ & 0.25 \\
$\{1,2\}$ & 0.3159 \\
$\{1,3\}$ & 0.3452 \\
$\{1,4\}$ & 0.4428 \\
$\{1,5\}$ & 0.3940 \\
$\{2,3\}$ & 0.3646 \\
$\{2,4\}$ & 0.4618 \\
$\{2,5\}$ & 0.4132 \\
$\{3,4\}$ & 0.4904 \\
$\{3,5\}$ & 0.4420 \\
$\{4,5\}$ & 0.5380 \\
$\{1,2,3\}$ & 0.5058 \\
$\{1,2,4\}$ & 0.6008 \\
$\{1,2,5\}$ & 0.5523 \\
$\{1,3,4\}$ & 0.6286 \\
$\{1,3,5\}$ & 0.5814 \\
$\{1,4,5\}$ & 0.6751 \\
$\{2,3,4\}$ & 0.6471 \\
$\{2,3,5\}$ & 0.5999 \\
$\{2,4,5\}$ & 0.6934 \\
$\{3,4,5\}$ & 0.7208 \\
$\{1,2,3,4\}$ & 0.7815 \\
$\{1,2,3,5\}$ & 0.7356 \\
$\{1,2,4,5\}$ & 0.8267 \\
$\{1,3,4,5\}$ & 0.8712 \\
$\{2,3,4,5\}$ & 0.8535 \\
$\{1,2,3,4,5\}$ & 1 \\
\hline &
\end{tabular}

Step 5. Rank the alternatives according to the scores of the alternatives; the greater the score of $S\left(a_{i}\right)$, the better the alternative $a_{i}$.

$$
\begin{aligned}
& S\left(a_{1}\right)=0.3366, \\
& S\left(a_{2}\right)=0.3799, \\
& S\left(a_{3}\right)=0.2934, \\
& S\left(a_{4}\right)=0.3017 .
\end{aligned}
$$

According to the scores of all the alternatives, the ranking is $a_{2}>a_{1}>a_{4}>a_{3}$. Thus the appropriate alternative is $a_{2}$.

\section{Comparison with Other Methods}

To demonstrate the validity of the proposed method in this paper, we apply the method to solve the same illustrative example by using the two existing MAGDM methods.
(1) Comparison with the Method Proposed by Tan [25]. Now, we use the approach to solve the investment problem (adopted from Tan [25]). Firstly, the IVIFEGC operator is utilized to aggregate the decision-making matrices into comprehensive evaluation matrix in Table 5.

Secondly, by using the proposed Euclidean distance based on Choquet integral, the distance and closeness coefficient of each alternative can be calculated.

$$
\begin{array}{r}
d\left(a_{1}, a^{*}\right)=0.6378, \\
d\left(a_{1}, a^{-}\right)=0.6180, \\
\xi\left(a_{1}\right)=0.4921 ; \\
d\left(a_{2}, a^{*}\right)=0.5950, \\
d\left(a_{3}, a^{-}\right)=0.6454, \\
\xi\left(a_{2}\right)=0.5203 ; \\
d\left(a_{3}, a^{*}\right)=0.6177, \\
d\left(a_{3}, a^{-}\right)=0.6679, \\
\xi\left(a_{3}\right)=0.5195 ; \\
d\left(a_{5}, a^{*}\right)=0.6273, \\
\left.d\left(a_{5}, a^{-}\right)=0.6173, a^{*}\right)=0.6318, \\
\xi\left(a_{4}, a^{-}\right)=0.6357, \\
\xi\left(a_{4}\right)=0.5015 ;
\end{array}
$$

Finally, according to the closeness coefficients of alternatives, the raking is $a_{2}>a_{3}>a_{4}>a_{5}>\alpha_{1}$. The result is the same as Tan [25].

(2) Comparison with the Method Proposed by Liu and Li [34]. To further demonstrate the validity of the proposed methods in this paper, we solve the same illustrative example proposed in Liu and $\mathrm{Li}$ [34]. To facilitate the calculation, we give the fuzzy measure of the individual expert: $g\left(e_{1}\right)=0.35, g\left(e_{2}\right)=$ 0.40 , and $g\left(e_{3}\right)=0.35$. Meanwhile, the fuzzy measure of the criterion is given: $g\left(a_{1}\right)=0.61, g\left(a_{2}\right)=0.59$, and $g\left(a_{3}\right)=$ 0.58 . By using the proposed method of this paper, the ranking result is listed in Table 6.

From Table 6, we can see that the proposed method has the same ranking result as the method proposed by Liu and Li [34]. From the result, we can see that the overall values of $r_{i}(i=1,2,3,4)$ using IVIFEGC operator are bigger than 
TABLE 3: Comprehensive evaluation matrix.

\begin{tabular}{ccccc}
\hline & $a_{1}$ & $a_{2}$ & $a_{3}$ & $a_{4}$ \\
\hline$c_{1}$ & $([0.5562,0.6687]$, & $([0.5380,0.6384]$, & $([0.6433,0.7888]$, & $([0.5310,0.6320]$, \\
& $[0.1506 .0 .2434])$ & $[0.1267,0.2845])$ & $[0.1000,0.2000])$ & $[0.1726,0.2958])$ \\
$c_{2}$ & $([0.5770,0.6908]$, & $([0.5740,0.7042]$, & $([0.5288,0.6291]$, & $([0.4862,0.6104]$, \\
& $[0.1451,0.2454])$ & $[0.1513,0.2516])$ & $[0.1221,0.2705])$ & $[0.1600,0.2606])$ \\
$c_{3}$ & $([0.5188,0.6353]$, & $([0.4955,0.6145]$, & $([0.5162,0.6164]$, & $([0.4510,0.5996]$, \\
& $[0.1405,0.2554])$ & $[0.2072,0.3082])$ & $[0.1712,0.2943])$ & $(0.1653,0.2808])$ \\
$c_{4}$ & $([0.4793,0.6055]$, & $([0.5440,0.7086]$, & $([0.5422,0.6572]$, & $([0.5675,0.7142]$, \\
& $[0.1916,0.2957])$ & $[0.1000,0.2000])$ & $[0.1658,0.2661])$ & $[0.1688,0.2690])$ \\
$c_{5}$ & $([0.3454,0.4917]$, & $([0.4129,0.5000]$, & $([0.2146,0.3154]$, & $([0.3448,0.4497]$, \\
& $[0.2643,0.3835])$ & $[0.2323,0.3331])$ & $[0.5280,0.6287])$ & $[0.3109,0.4735])$ \\
\hline
\end{tabular}

TABLE 4: The fuzzy measure of criterion and the subset of the criteria.

\begin{tabular}{lc}
\hline$c_{i}$ & $g\left(c_{i}\right)$ \\
\hline$\{1\}$ & 0.30 \\
$\{2\}$ & 0.25 \\
$\{3\}$ & 0.25 \\
$\{4\}$ & 0.3 \\
$\{5\}$ & 0.35 \\
$\{1,2\}$ & 0.5013 \\
$\{1,3\}$ & 0.5013 \\
$\{1,4\}$ & 0.5415 \\
$\{1,5\}$ & 0.5818 \\
$\{2,3\}$ & 0.4594 \\
$\{2,4\}$ & 0.5013 \\
$\{2,5\}$ & 0.5431 \\
$\{3,4\}$ & 0.5013 \\
$\{3,5\}$ & 0.5431 \\
$\{4,5\}$ & 0.5818 \\
$\{1,2,3\}$ & 0.6698 \\
$\{1,2,4\}$ & 0.7035 \\
$\{1,2,5\}$ & 0.7372 \\
$\{1,3,4\}$ & 0.7035 \\
$\{1,3,5\}$ & 0.7372 \\
$\{1,4,5\}$ & 0.7683 \\
$\{2,3,4\}$ & 0.6698 \\
$\{2,3,5\}$ & 0.7049 \\
$\{2,4,5\}$ & 0.7372 \\
$\{3,4,5\}$ & 0.7372 \\
$\{1,2,3,4\}$ & 0.8392 \\
$\{1,2,3,5\}$ & 0.8674 \\
$\{1,2,4,5\}$ & 0.8935 \\
$\{1,3,4,5\}$ & 0.8935 \\
$\{2,3,4,5\}$ & 0.8674 \\
\hline & 1 \\
$\{1,3,4,5\}$ &
\end{tabular}

those obtained using IVIFWPGBM operator. That is to say that the proposed IVIFEGC operator shows more optimistic attitude of decision-makers than the IVIFWPGBM operator in aggregation process.
From above two comparisons with other operators, they have the same ranking results as the proposed method. This can verify that the IVIFEGC operator is also an effective approach for decision-making information aggregation.

\section{Conclusion}

In order to solve the multiattribute decision-making problem in which the attributes are interdependent under the intervalvalued intuitionistic fuzzy environment, by using geometric Choquet integral and Einstein operation laws for fuzzy sets, we developed a new interval-valued fuzzy Einstein geometric Choquet integral operator to aggregate the interdependent decision-making information. Based on the study of the properties of the operator, the decision-making approach based on the operator is proposed. Finally, an illustrative example is given to illustrate the multicriteria group decisionmaking process, and with the comparison with previous approaches, the effectiveness of the method is demonstrated. The paper has provided a new calculation law, which is Einstein operation laws, to solve the multicriteria group decisionmaking problems where the attributes are interdependent under the interval-valued intuitionistic fuzzy environment. In later research, we can extend the developed operator and apply it to other intuitionistic fuzzy environments, such as triangle intuitionistic fuzzy environment and trapezoid fuzzy environment. In addition, we will also develop some real applications of the proposed operator in actual areas, such as supply chain risk evaluation and strategic logistics service supplier selection evaluation in cross-border e-commerce.

\section{Conflicts of Interest}

The authors declare that there are no conflicts of interest regarding the publication of this paper.

\section{Acknowledgments}

This paper is supported by Research Project of Philosophy and Social Science of Zhejiang Province (no. 18NDJC283YB), Natural Science Foundation of Zhejiang Province (no. LY17G030002), Soft Science Project of Ningbo 
TABLE 5: Comprehensive evaluation matrix.

\begin{tabular}{ccccc}
\hline & $c_{1}$ & $c_{2}$ & $c_{3}$ & $c_{4}$ \\
\hline$a_{1}$ & $([0.3062,0.4702]$, & $([0.4381,0.5658]$, & $([0.2499,0.4738]$, & $([0.3305,0.5775]$, \\
& $[0.2613,0.3601])$ & $[01273,0.2960])$ & $[0.3199,0.2275])$ & $[0.1883,0.3901])$ \\
$a_{2}$ & $([0.3553,0.5428]$, & $([0.4403,0.6722]$, & $([0.4254,0.6772]$, & $([0.5317,0.7109]$, \\
& $[0.2841,0.3867])$ & $[0.1674,0.2960])$ & $[0.1273,0.2275])$ & $(0.1333,0.2606])$ \\
$a_{3}$ & $([0.5719,0.7109]$, & $([0.5735,0.6767]$, & $([0.5000,0.6390]$, & $([0.2820,0.4384]$, \\
& $[0.1555,0.2565])$ & $[0.1555,0.2565])$ & $[0.1674,0.3000])$ & $(0.3199,0.4593])$ \\
$a_{4}$ & $([0.3248,0.5021]$, & $([0.5020,0.7109]$, & $([0.1970,0.4312]$, & $([0.3373,0.7000]$, \\
& $[0.2275,0.3969])$ & $[0.1273,0.2606])$ & $[0.2221,0.3868])$ & $[0.1000,0.2000])$ \\
$a_{5}$ & $([0.6390,0.7394]$, & $([0.4738,0.6099]$, & $([0.5000,0.6390]$, & $([0.4738,0.5775]$, \\
& $[0.1273,0.2606])$ & $[0.1678,0.2954])$ & $[0.2000,0.3000])$ & $[0.2499,0.4225])$ \\
\hline
\end{tabular}

TABLE 6: Comparisons of ranking results from different methods.

\begin{tabular}{lcc}
\hline Method & The proposed method & $\begin{array}{c}\text { Liu and Li's } \\
\text { method [34] }\end{array}$ \\
\hline Score functions & $s f\left(\widetilde{r}_{1}\right)=-0.0891$ & $s f\left(\widetilde{r}_{1}\right)=-0.1087$ \\
& $s f\left(\widetilde{r}_{2}\right)=-0.0545$ & $s f\left(\widetilde{r}_{2}\right)=-0.0756$ \\
Ranking & $s f\left(\widetilde{r}_{3}\right)=0.1504$ & $s f\left(\widetilde{r}_{3}\right)=0.0514$ \\
& $s f\left(\widetilde{r}_{4}\right)=0.2139$ & $s f\left(\widetilde{r}_{4}\right)=0.1448$ \\
& $z_{4}>z_{3}>z_{2}>z_{1}$ & $z_{4}>z_{3}>z_{2}>z_{1}$ \\
\hline
\end{tabular}

(no. 2017A10068), and the Key Research Institute of Philosophy and Social Science of Zhejiang Province (Modern Port Service Industry and Creative Culture Research Center) (no. 15JDLG01YB).

\section{References}

[1] K. T. Atanassov, "Intuitionistic fuzzy sets," Fuzzy Sets and Systems, vol. 20, no. 1, pp. 87-96, 1986.

[2] L. A. Zadeh, "Fuzzy sets," Information and Control, vol. 8, no. 3, pp. 338-353, 1965.

[3] D. Yu and S. Shi, "Researching the development of Atanassov intuitionistic fuzzy set: using a citation network analysis," Applied Soft Computing, vol. 32, pp. 189-198, 2015.

[4] D. Yu and H. Liao, "Visualization and quantitative research on intuitionistic fuzzy studies," Journal of Intelligent \& Fuzzy Systems: Applications in Engineering and Technology, vol. 30, no. 6, pp. 3653-3663, 2016.

[5] D. Yu, "A scientometrics review on aggregation operator research," Scientometrics, vol. 105, no. 1, pp. 115-133, 2015.

[6] K. Atanassov and G. Gargov, "Interval valued intuitionistic fuzzy sets," Fuzzy Sets and Systems, vol. 31, no. 3, pp. 343-349, 1989.

[7] D. Yu, "Decision making based on generalized geometric operator under interval-valued intuitionistic fuzzy environment," Journal of Intelligent \& Fuzzy Systems: Applications in Engineering and Technology, vol. 25, no. 2, pp. 471-480, 2013.

[8] H. Zhao, Z. S. Xu, M. F. Ni, and S. S. Liu, "Generalized aggregation operators for intuitionistic fuzzy sets," International Journal of Intelligent Systems, vol. 25, no. 1, pp. 1-30, 2010.

[9] J. Lin and Q. Zhang, "Some continuous aggregation operators with interval-valued intuitionistic fuzzy information and their application to decision making," International Journal of Uncertainty, Fuzziness and Knowledge-Based Systems, vol. 20, no. 2, pp. 185-209, 2012.
[10] G. Wei, "Some induced geometric aggregation operators with intuitionistic fuzzy information and their application to group decision making," Applied Soft Computing, vol. 10, no. 2, pp. 423-431, 2010.

[11] D. Yu, Y. Wu, and T. Lu, "Interval-valued intuitionistic fuzzy prioritized operators and their application in group decision making," Knowledge-Based Systems, vol. 30, pp. 57-66, 2012.

[12] $\mathrm{H}$. Wu and X. Su, "Interval-valued intuitionistic fuzzy prioritized hybrid weighted aggregation operator and its application in decision making," Journal of Intelligent \& Fuzzy Systems: Applications in Engineering and Technology, vol. 29, no. 4, pp. 1697-1709, 2015.

[13] W. Zhou and J. M. He, "Interval-valued intuitionistic fuzzy ordered precise weighted aggregation operator and its application in group decision making," Technological and Economic Development of Economy, vol. 20, no. 4, pp. 648-672, 2014.

[14] H. Zhao and Z. Xu, "Group decision making with density-based aggregation operators under interval-valued intuitionistic fuzzy environments," Journal of Intelligent \& Fuzzy Systems: Applications in Engineering and Technology, vol. 27, no. 2, pp. 1021-1033, 2014.

[15] Z. Xu and X. Gou, "An overview of interval-valued intuitionistic fuzzy information aggregations and applications," Granular Computing, vol. 2, no. 1, pp. 13-39, 2017.

[16] M. M. Xia, Z. S. Xu, and B. Zhu, "Some issues on intuitionistic fuzzy aggregation operators based on Archimedean t-conorm and t-norm," Knowledge-Based Systems, vol. 31, pp. 78-88, 2012.

[17] W. Wang and X. Liu, "Interval-valued intuitionistic fuzzy hybrid weighted averaging operator based on Einstein operation and its application to decision making," Journal of Intelligent \& Fuzzy Systems: Applications in Engineering and Technology, vol. 25, no. 2, pp. 279-290, 2013.

[18] W. Z. Wang and X. W. Liu, "The multi-attribute decision making method based on interval-valued intuitionistic fuzzy Einstein hybrid weighted geometric operator," Computers \& Mathematics with Applications, vol. 66, no. 10, pp. 1845-1856, 2013.

[19] Y. R. Yang and S. Yuan, "Induced interval-valued intuitionistic fuzzy Einstein ordered weighted geometric operator and their application to multiple attribute decision making," Journal of Intelligent \& Fuzzy Systems: Applications in Engineering and Technology, vol. 26, no. 6, pp. 2945-2954, 2014.

[20] X. Cai and L. Han, "Some induced Einstein aggregation operators based on the data mining with interval-valued intuitionistic fuzzy information and their application to multiple attribute decision making," Journal of Intelligent \& Fuzzy Systems: Applications in Engineering and Technology, vol. 27, no. 1, pp. 331-338, 2014. 
[21] M. Grabisch, "Fuzzy integral in multicriteria decision making," Fuzzy Sets and Systems, vol. 69, no. 3, pp. 279-298, 1995.

[22] V. Torra, "Information fusion in data mining," Journal of Optimization Theory \& Applications, vol. 133, no. 3, pp. 371-383, 2003.

[23] G. Choquet, “Theory of capacities," Annales de l'Institut Fourier, vol. 5, pp. 131-295, 1954.

[24] Z. Xu, "Choquet integrals of weighted intuitionistic fuzzy information," Information Sciences, vol. 180, no. 5, pp. 726-736, 2010.

[25] C. Q. Tan, "A multi-criteria interval-valued intuitionistic fuzzy group decision making with Choquet integral-based TOPSIS," Expert Systems with Applications, vol. 38, no. 4, pp. 3023-3033, 2011.

[26] C. Tan and X. Chen, "Interval-valued intuitionistic fuzzy multicriteria group decision making based on VIKOR and choquet integral," Journal of Applied Mathematics, vol. 2013, Article ID 656879, 16 pages, 2013.

[27] Z. Xu and M. Xia, "Induced generalized intuitionistic fuzzy operators," Knowledge-Based Systems, vol. 24, no. 2, pp. 197-209, 2011.

[28] F. Meng, Q. Zhang, and J. Zhan, "The interval-valued intuitionistic fuzzy geometric choquet aggregation operator based on the generalized banzhaf index and 2-additive measure," Technological and Economic Development of Economy, vol. 21, no. 2, pp. 186-215, 2015.

[29] H. Cheng and J. Tang, "Interval-valued intuitionistic fuzzy multi-criteria decision making based on the generalized Shapley geometric Choquet integral," Journal of Industrial and Production Engineering, vol. 33, no. 1, pp. 1-16, 2016.

[30] X. Gu, P. Zhao, and Y. Wang, "Models for multiple attribute decision making based on the Einstein correlated aggregation operators with interval-valued intuitionistic fuzzy information," Journal of Intelligent \& Fuzzy Systems: Applications in Engineering and Technology, vol. 26, no. 4, pp. 2047-2055, 2014.

[31] P. D. Liu, "Multiple attribute group decision making method based on interval-valued intuitionistic fuzzy power heronian aggregation operators," Computers \& Industrial Engineering, vol. 108, pp. 199-212, 2017.

[32] P. Liu and S.-M. Chen, "Group Decision Making Based on Heronian Aggregation Operators of Intuitionistic Fuzzy Numbers," IEEE Transactions on Cybernetics, 2016.

[33] P. D. Liu, S. M. Chen, and J. Liu, "Some intuitionistic fuzzy interaction partitioned Bonferroni mean operators and their application to multi-attribute group decision making," Information Sciences, vol. 411, pp. 98-121, 2017.

[34] P. Liu and H. Li, "Interval-Valued Intuitionistic Fuzzy Power Bonferroni Aggregation Operators and Their Application to Group Decision Making," Cognitive Computation, pp. 1-19, 2017.

[35] P. D. Liu, L. He, and X. C. Yu, "Generalized hybrid aggregation operators based on the 2-dimension uncertain linguistic information for multiple attribute group decision making," Group Decision and Negotiation, vol. 25, no. 1, pp. 103-126, 2016.

[36] P. Liu and P. Wang, "Some improved linguistic intuitionistic fuzzy aggregation operators and their applications to multipleattribute decision making," International Journal of Information Technology \& Decision Making, vol. 16, no. 3, pp. 817-850, 2017.

[37] P. Liu and L. Shi, "Some neutrosophic uncertain linguistic number Heronian mean operators and their application to multi-attribute group decision making," Neural Computing and Applications, vol. 28, no. 5, pp. 1079-1093, 2017.
[38] P. Liu, L. Zhang, X. Liu, and P. Wang, "Multi-valued neutrosophic number bonferroni mean operators with their applications in multiple attribute group decision making," International Journal of Information Technology \& Decision Making, vol. 15, no. 5, pp. 1181-1210, 2016.

[39] P. Liu and G. Tang, "Multi-criteria Group Decision-Making Based on Interval Neutrosophic Uncertain Linguistic Variables and Choquet Integral," Cognitive Computation, vol. 8, no. 6, pp. 1036-1056, 2016.

[40] P. D. Liu, J. L. Liu, and J. M. Merigó, "Partitioned Heronian means based on linguistic intuitionistic fuzzy numbers for dealing with multi-attribute group decision making," Applied Soft Computing, vol. 62, pp. 395-422, 2018, https://doi.org/10.1016/ j.asoc.2017.10.017.

[41] P. Liu, J. Liu, and S.-M. Chen, "Some intuitionistic fuzzy Dombi Bonferroni mean operators and their application to multiattribute group decision making," Journal of the Operational Research Society, 2017.

[42] D. Liu, X. H. Chen, and D. Peng, "Interval-valued intuitionistic fuzzy ordered weighted cosine similarity measure and its application in investment decision-making," Mathematical Problems in Engineering, vol. 2017, Article ID 1891923, 11 pages, 2017.

[43] R. Krishankumar, K. S. Ravichandran, and R. Ramprakash, "A scientific decision framework for supplier selection under interval valued intuitionistic fuzzy environment," Mathematical Problems in Engineering, vol. 2017, Article ID 1438425, 18 pages, 2017.

[44] M. Sugeno, Theory of Fuzzy Integral and Its Application, Tokyo Institute of Technology, Tokyo, Japan, 2017. 


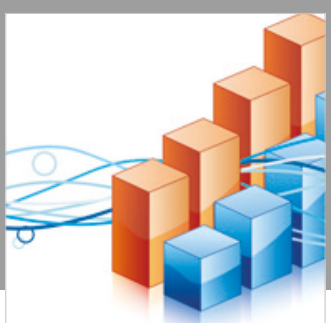

Advances in

Operations Research

\section{-n-m}
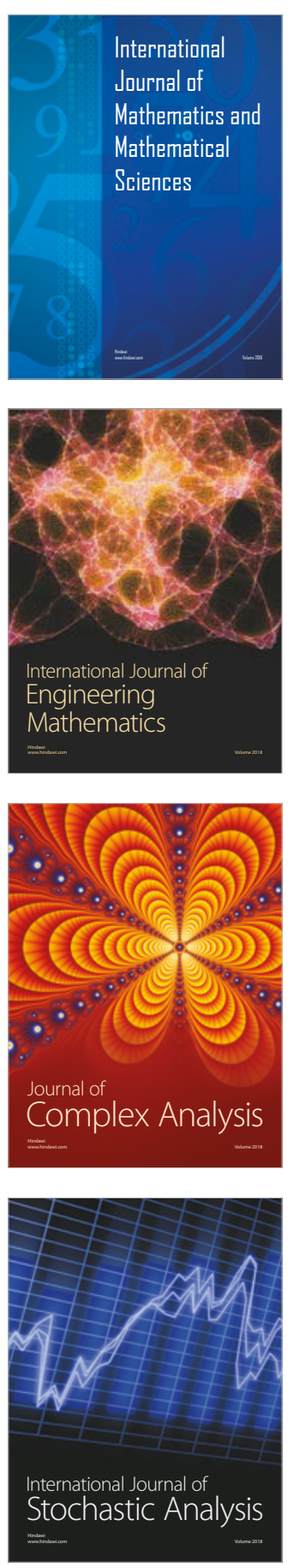
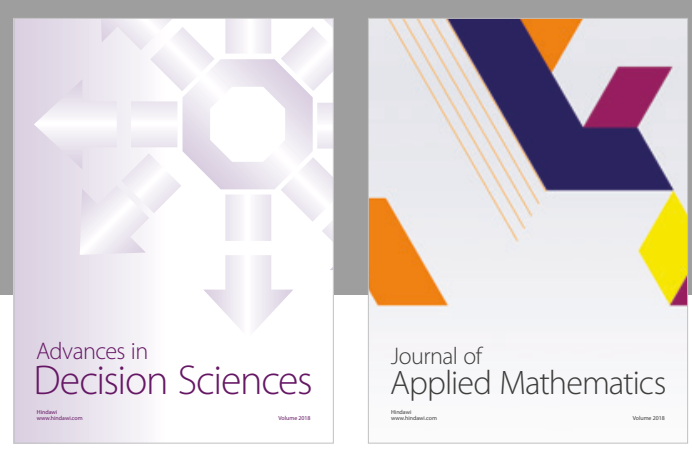

Journal of

Applied Mathematics
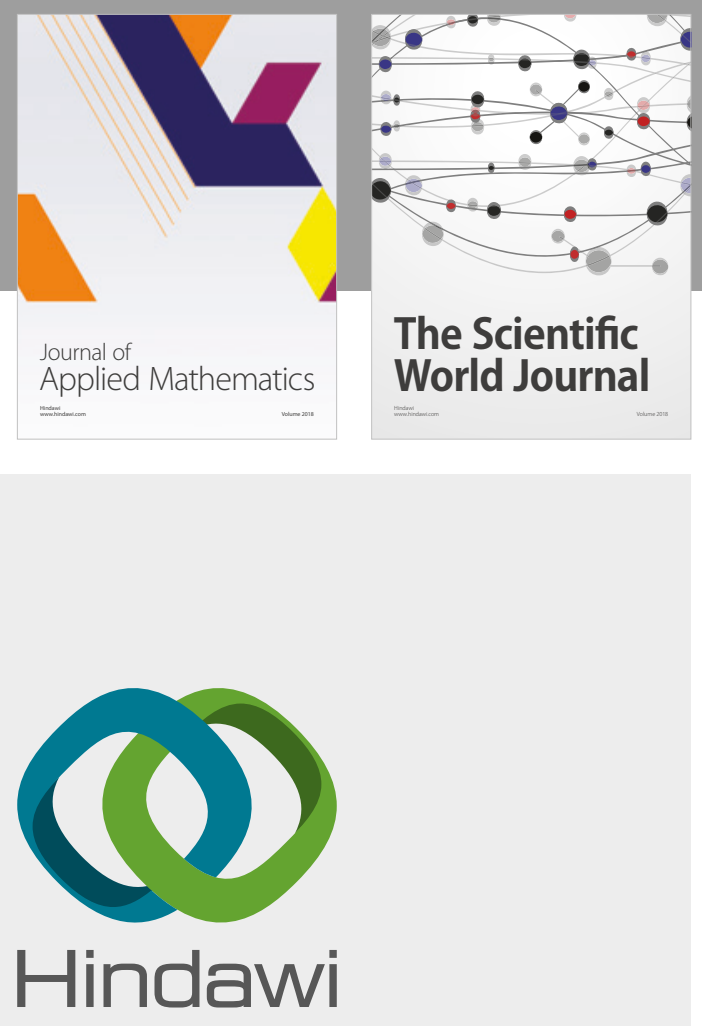

Submit your manuscripts at

www.hindawi.com

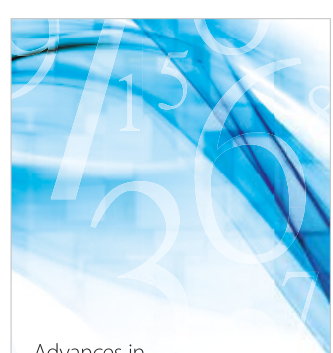

Advances in
Numerical Analysis
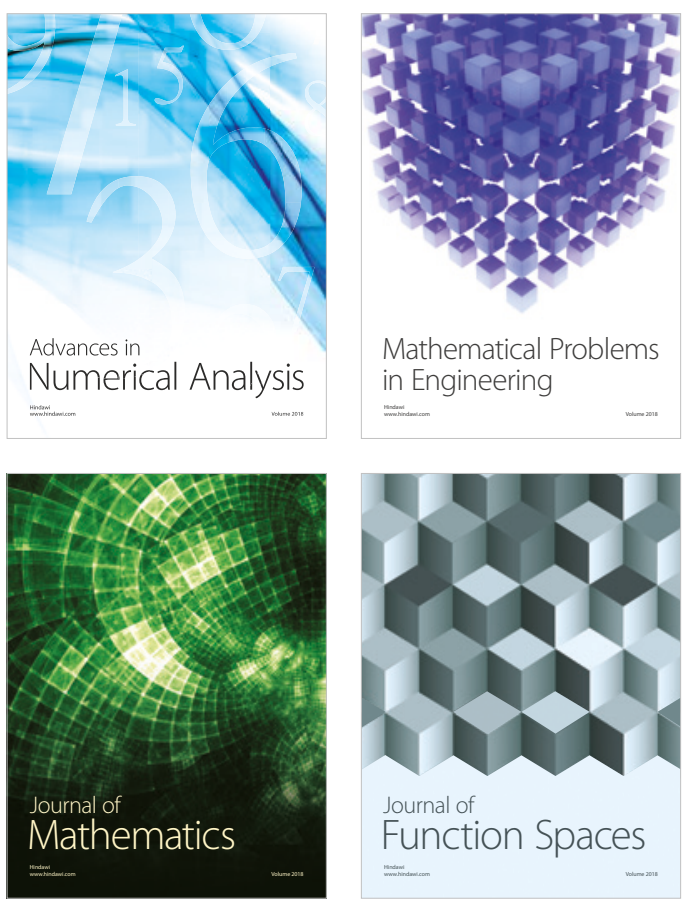

Mathematical Problems in Engineering

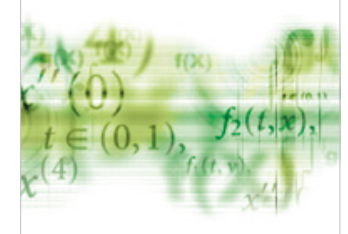

International Journal of

Differential Equations

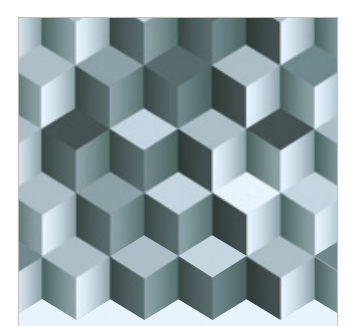

Journal of

Function Spaces

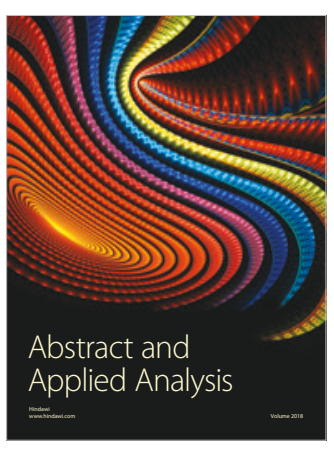

The Scientific

World Journal

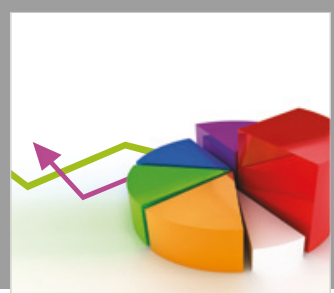

Journal of

Probability and Statistics
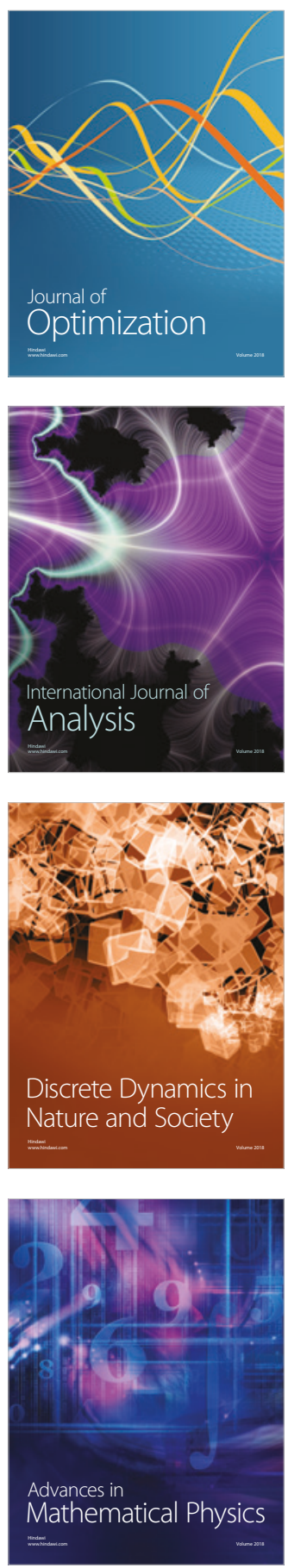